Artículos originales

\title{
Relación del riesgo nutricional y la presencia de depresión en adultos mayores ambulatorios de Aguascalientes, México
}

\author{
Relationship of the nutritional risk and the \\ presence of depression in elderly in Aguascalientes, \\ Mexico
}

Jiménez-Saldívar, P; Pedroza-García, KA; Ramírez-Orozco, RE

\section{P Jiménez-Saldívar}

Universidad Autónoma de Aguascalientes, México KA Pedroza-García

Universidad Autónoma de Aguascalientes, México

RE Ramírez-Orozco dcmrero@gmail.com

Universidad Autónoma de Aguascalientes, México

\section{Lux Médica}

Universidad Autónoma de Aguascalientes, México

ISSN: 2007-1655

Periodicidad: Cuatrimestral

vol. 17, núm. 49, 2022

luxmedica.editorial@gmail.com

Recepción: 01 Octubre 2021

Aprobación: 10 Noviembre 2021

URL:

https://revistas.uaa.mx/index.php/luxmedica/article/view/3373

DOI:

https://doi.org/10.33064/491m20223373

\section{(i) (2)}

Esta obra está bajo una Licencia Creative Commons AtribuciónNoComercial-CompartirIgual 4.0 Internacional.
Resumen: Introducción: La depresión es un trastorno afectivo caracterizado por sentimientos de desesperación y tristeza. Existe una relación entre la malnutrición y la presencia de depresión en el adulto mayor institucionalizado y/o de comunidad rural. Objetivo: estudiar la relación de la depresión y el estado nutricional en el adulto mayor de comunidad de Aguascalientes, México. Métodos: Estudio descriptivo correlacional, transversal, los participantes fueron adultos mayores ( $>60$ años) pertenecientes a una estancia de día, en Aguascalientes, México $(n=50)$. El estado nutricional se evaluó mediante el Mini Nutritional Assessment (MNA) y antropometría. La depresión se evaluó mediante la Escala de Depresión Geriátrica de Yesavage (GDS). Se utilizaron pruebas estadísticas como: U de Mann Whitney para diferencias entre grupos y la asociación entre riesgo nutricional y depresión por Correlación de Pearson. Resultados: La clasificación del estado nutricional de los adultos mayores evaluados fue la siguiente: Normalidad, $76 \%$; en riesgo de malnutrición, 22\%; malnutrición, 2\%. La prevalencia de depresión fue de $40 \%$. Los indicadores antropométricos se encuentran alterados en el adulto mayor con depresión ( $\mathrm{p}=0.03$ ). Se observó una correlación inversa entre el MNA y la EDG $(\mathrm{r}=-0.528, \mathrm{p}=0.0001)$ y un menor peso o IMC. Esto, aunado a una mayor edad, contribuirá a la presencia de depresión. Conclusión: El riesgo nutricional es un factor importante a considerar en la presencia de depresión en el adulto mayor. Esta relación es una pauta a seguir como parte del tratamiento nutricional de esta población como adyuvante en la terapia del adulto mayor con depresión.

Palabras clave: depresión, riesgo nutricio, mini nutritional assessment, adulto mayor.

Abstract: Introduction: Depression is a mood disorder characterized by feelings of despair and sadness. There is a relationship between malnutrition and the presence of depression in institutionalized or rural community elderly patients. Objective: determine the relationship of depression and nutritional status in elderly community people. Methods: A crosssectional analytical study, participants were institutionalized 


\begin{abstract}
older adults in Aguascalientes, Mexico $(n=50)$. The nutritional status was assessed by Mini Nutritional Assessment (MNA) and anthropometry. Depression was determined using the Geriatric Depression Scale of Yesavage (GDS). Statistic tests used for analysis: Mann Whitney $U$ test for differences between groups and association between nutritional risk and depression by Pearson's correlation coefficient. Results: Nutritional status of older adults was evaluated and obtained the following results: Normal: $76 \%$, malnutrition risk: $22 \%$, malnutrition: $2 \%$. Depression prevalence was $40 \%$. Anthropometric measures are altered in older adults with depression $(\mathrm{p}=0.03)$, an inverse correlation was observed between MNA and GDS, $(r=-0.528, p$ $=0.0001$ ), a minor weight or BMI and a higher age will contribute to depression presence. Conclusion: The nutritional risk is an important factor to consider in the presence of depression in the elderly. Being this relationship a pattern to follow as part of the nutritional treatment of this population as an adjuvant in the treatment of older adults with depression.
\end{abstract}

Keywords: Depression, Nutritional Status, Mini Nutritional Assessment, Elderly.

\title{
INTRODUCCIÓN
}

De acuerdo con estudios de la Organización Mundial de la Salud (OMS), entre el año 2015 y 2050 la proporción de personas mayores de 60 años pasará de 900 millones a 2,000 millones, aumentando de un $12 \%$ a un $22 \%$. En México, la población de adultos mayores representa el 8.9\% (10\#055,379) de la población total, siendo una prevalencia similar en el estado de Aguascalientes $(9 \%=119,691){ }^{1,2}$

Por otro lado, la depresión es un trastorno afectivo caracterizado por la presencia de sentimientos de desesperación y tristeza, así como falta de energía o cansancio constante, siendo frecuentes estos síntomas durante un periodo de al menos de tres semanas. La presencia de estos factores establecerá un diagnóstico de depresión, de acuerdo con los criterios del Manual diagnóstico y estadístico de los trastornos mentales (DSM-V). ${ }^{3}$ Según estudios de Sotelo y cols., es el trastorno afectivo más frecuente en personas mayores de 60 años, reportando una prevalencia de entre el $15 \%$ y el $20 \%$ en la población ambulatoria y del $25 \%$ al $40 \%$ en el paciente hospitalizado. ${ }^{4}$

La depresión en el adulto mayor está conformada de manera heterogénea; es decir, los factores biológicos, la coexistencia de patologías y el uso de múltiples medicamentos, aunado a factores psicosociales, tienen un papel importante en la aparición de la sintomatología depresiva. ${ }^{5}$ Actualmente existen diversos instrumentos de evaluación de trastornos mentales, lo que facilita el diagnóstico de probables casos de depresión. ${ }^{6}$ La Escala de Depresión Geriátrica de Yesavage (GDS) identifica los problemas característicos que presenta este grupo de edad que tiene sintomatología depresiva., $7,9,10$

Otra de las problemáticas más frecuentes del adulto mayor es la desnutrición, la cual repercute de manera importante en el estado de salud. Existe una relación directa con el aumento de riesgo a desarrollar otras morbilidades y esto, a su 
vez, conlleva un riesgo de mortalidad, debido causas como anemia, respuesta inmunológica deficiente a infecciones, retraso en la cicatrización de heridas y agravamiento de la enfermedad de base. Algunos de los factores que favorecen la desnutrición del adulto mayor incluyen trastornos de deglución, xerostomía, anorexia, enfermedades crónicas y las enfermedades mentales. ${ }^{11}$

En México, el aumento de la desnutrición y la disminución de la obesidad en esta etapa de la vida puede explicarse por la presencia de comorbilidades asociadas a la obesidad, las cuales incrementan la mortalidad de manera importante. Incluso se ha reportado que aquellos individuos con un peso e IMC adecuado o ligeramente mayor tienen mejor pronóstico en algunas enfermedades. La aparición de enfermedades crónico degenerativas, el deterioro fisiológico, la carencia de protección social y redes de apoyo económico favorecen la aparición de la desnutrición. ${ }^{12,13}$

Con el fin de prevenir o detectar a los sujetos en riesgo de desnutrición, se han creado instrumentos de tamizaje. Uno de los más conocidos es el Mini Nutritional Assessment (MNA), el cual consta de un cuestionario respecto a cambios en la alimentación, problemas de deglución y digestión, ingesta de líquidos, autonomía para alimentarse y variedad de la dieta, entre otros aspectos. De acuerdo al puntaje, se diagnosticará el estado nutricional; malnutrición: $<17$ puntos, riesgo nutricional: 17 a 23.5 puntos y estado nutricional normal: 24 a 30 puntos. Este es aplicado por médicos, personal de enfermería y principalmente por nutriólogos. ${ }^{6,14}$

La presencia de depresión en el adulto mayor se ha relacionado con una mayor probabilidad de sufrir alguna enfermedad, como lo observado por Kronfly, quién demostró que los pacientes depresivos tienen casi cuatro veces más probabilidades de sufrir hipertensión arterial o cáncer, mayor ansiedad, así como también presentar un estado nutricional desfavorable. ${ }^{15}$ También se relaciona la pérdida de apetito y la consecuente pérdida de peso. ${ }^{16,17}$

El objetivo del presente estudio fue evaluar la presencia de depresión en el adulto mayor y determinar la asociación con el estado de nutrición y otras comorbilidades.

\section{MATERIAL Y MÉTODOS}

\section{Tipo de estudio y sujetos}

Estudio descriptivo correlacional, transversal, en el que participaron hombres y mujeres mayores de 60 años $(n=50)$ que acudían a una estancia para adultos mayores aparentemente sanos o sin morbilidades incapacitantes, en la ciudad de Aguascalientes. La muestra fue determinada y obtenida a conveniencia. El presente estudio fue sometido y aceptado por un comité de investigación para la verificación de las consideraciones éticas de dicho proyecto. De todos los participantes del estudio se obtuvo su consentimiento informado. 


\section{Caracteristicas antropométricas}

Las mediciones antropométricas incluyeron: peso y talla, estos fueron evaluados sin calzado, joyería y la menor cantidad de ropa posible. La medición la llevó a cabo una nutrióloga certificada. A partir de las mediciones de peso y talla, se determinó el IMC. Todas las mediciones fueron evaluadas por la misma persona, además de evaluarse a los participantes bajo las mismas condiciones y tiempo. El peso $(\mathrm{kg})$ fue valorado en una báscula SECA ${ }^{\circ}$ modelo 700 con capacidad de $160 \mathrm{~kg}$. La talla $(\mathrm{cm})$ fue determinada en el estadímetro de dicha báscula, estando el sujeto de pie y descalzo y con el mínimo de ropa. El índice de masa corporal (IMC) fue interpretado de acuerdo a los valores establecidos por los criterios de la OMS.

La evaluación de riesgo nutricional se realizó mediante la aplicación del Mini Nutritional Assessment (MNA). La puntuación global del MNA es de 30 puntos, cuya interpretación es la siguiente: menor a 17 puntos, existe una malnutrición; un puntaje de 17 a 23.5, se tiene riesgo nutricional, y un puntaje final mayor a 24 , se registra un estado nutricional adecuado. Esta prueba ha demostrado su eficacia y precisión en la valoración del estado nutricional en población geriátrica con una sensibilidad del 96\%, especificidad del 98\% y con un valor predictivo del $97 \%{ }^{14}$

\section{Presencia de depresión}

Para evaluar la presencia de depresión, se aplicó la Escala de Depresión Geriátrica de Yesavage (GDS) en su versión original de 30 preguntas. Su interpretación es la siguiente: $0-10$ puntos se considera sin depresión; el punto de corte de $\geq$ 11 indica que la persona presenta depresión, con una sensibilidad del $84 \%$ y una especificidad del $95 \%$, y, finalmente, un puntaje $>14$ se considera depresión mayor.

\section{Análisis estadistico}

Se realizó un análisis descriptivo. Para las variables cuantitativas, se calculó la media, la desviación estándar y el rango; las variables cualitativas se analizaron por frecuencias, proporciones y porcentajes. Se verificó la distribución de variables cuantitativas mediante la prueba de Kolmogorov-Smirnov. Para el análisis inferencial se utilizaron las siguientes pruebas: T-Student para muestras independientes y la prueba de Correlación de Pearson; se consideró un nivel de significancia de $\mathrm{p}<0.05$. El análisis de los datos se llevo a cabo mediante el programa SPSS versión 20.

\section{RESULTADOS}

En total, participaron 50 adultos mayores, 31 mujeres (62\%) y 19 hombres (38\%), y la edad promedio de los participantes fue de $78 \pm 6.3$ años. En cuanto a la presencia de enfermedad crónico degenerativa; la prevalencia fue la siguiente: HAS (62\%), DM2 (23\%), angina de pecho (5\%), enfermedad renal (4\%), 
enfermedades respiratorias (4\%) y otras enfermedades (2\%). Las características se muestran en la tabla 1.

\begin{tabular}{|c|c|c|c|}
\hline Variable & Mínimo & Máximo & Media \\
\hline Edad (años) & 65.0 & 91.0 & $78.1 \pm 6.3$ \\
\hline Talla $(\mathrm{cm})$ & 137.0 & 178.0 & $154.5 \pm 9.8$ \\
\hline Peso $(\mathrm{Kg})$ & 44.6 & 106.0 & $65.2 \pm 12.3$ \\
\hline $\operatorname{IMC}\left(\mathrm{Kg} / \mathrm{m}^{2}\right)$ & 18.8 & 40.1 & $27.1 \pm 4.4$ \\
\hline Mini Nutritional Assessment (puntos) & 13.5 & 30.0 & $\begin{array}{c}25.2 \pm 3.2 \\
86+43\end{array}$ \\
\hline GDS (puntos) & 0.0 & 17.0 & \\
\hline
\end{tabular}

Tabla 1. Características generales de la muestra estudiada $(\mathrm{n}=50)$

Los datos son representados en medias, desviación estándar y rango, $(\mathrm{n}=50)$.

Estadística descriptiva. GDS: Escala de Depresión Geriátrica de Yesavage.

\section{Nutrición y su repercusión en el estado de ánimo}

De acuerdo con la prueba de cribado nutricional MNA el 22\% $(n=11)$ presenta riesgo de desnutrición y $2 \%(\mathrm{n}=1)$ desnutrición; para el restante $76 \%$ $(\mathrm{n}=38)$, el estado nutricional es normal. Por su parte, los resultados de la Escala de Depresión Geriátrica de Yesavage mostraron una mayor prevalencia sintomatología depresiva en $42 \%(n=21)$ de los participantes, y el $58 \%(n=29)$ se mostró sin depresión, siendo mayor en mujeres, $66 \%(\mathrm{n}=14)$, mientras que en hombres fue de $34 \%(n=7)$. Es menor la presencia de depresión en aquellos con peso adecuado y con puntaje en el cribado de entre 24 y 30 puntos (estado correcto de nutrición o sin riesgo), comparado con aquellos con un IMC por debajo de la normalidad (figura 1 y tabla 2).

\begin{tabular}{lccr}
\hline \multicolumn{1}{c}{ Variable } & Sin depresión $(n=21)$ & Con depresión $(n=29)$ & \multicolumn{1}{c}{$p$} \\
& & & \\
\hline Edad (años) & $76.4 \pm 5.4$ & $80.4 \pm 6.9$ & $\mathbf{0 . 0 3 3}$ \\
Talla (cm) & $178 \pm 10.5$ & $153.6 \pm 8.8$ & $\mathbf{0 . 5 5 9}$ \\
Peso $(\mathrm{Kg})$ & $67.5 \pm 11.0$ & $61.5 \pm 13.3$ & $\mathbf{0 . 0 3 4}$ \\
IMC $\left(\mathrm{Kg} / \mathrm{m}^{2}\right)$ & $28.1 \pm 4.2$ & $25.6 \pm 4.2$ & $\mathbf{0 . 0 3}$ \\
Mini Nutritional Assessment (puntos) & $26.6 \pm 1.8$ & $23 \pm 3.6$ & $\mathbf{0 . 0 0 0 1}$ \\
GDS (puntos) & $5.5 \pm 2.3$ & $13.1 \pm 1.8$ & $\mathbf{0 . 0 0 0 1}$ \\
\hline
\end{tabular}

Tabla 2. Antropometría y riesgo nutricional de acuerdo a la Escala de Depresión Geriátrica (GDS)

Prueba estadística utilizada: T-Student para muestras independientes; los datos son representados como media y desviación estándar. GDS: Escala de Depresión Geriátrica de Yesavage. Se consideró significativa con una $\mathrm{p}<0.05$; $(\mathrm{n}=50)$. 

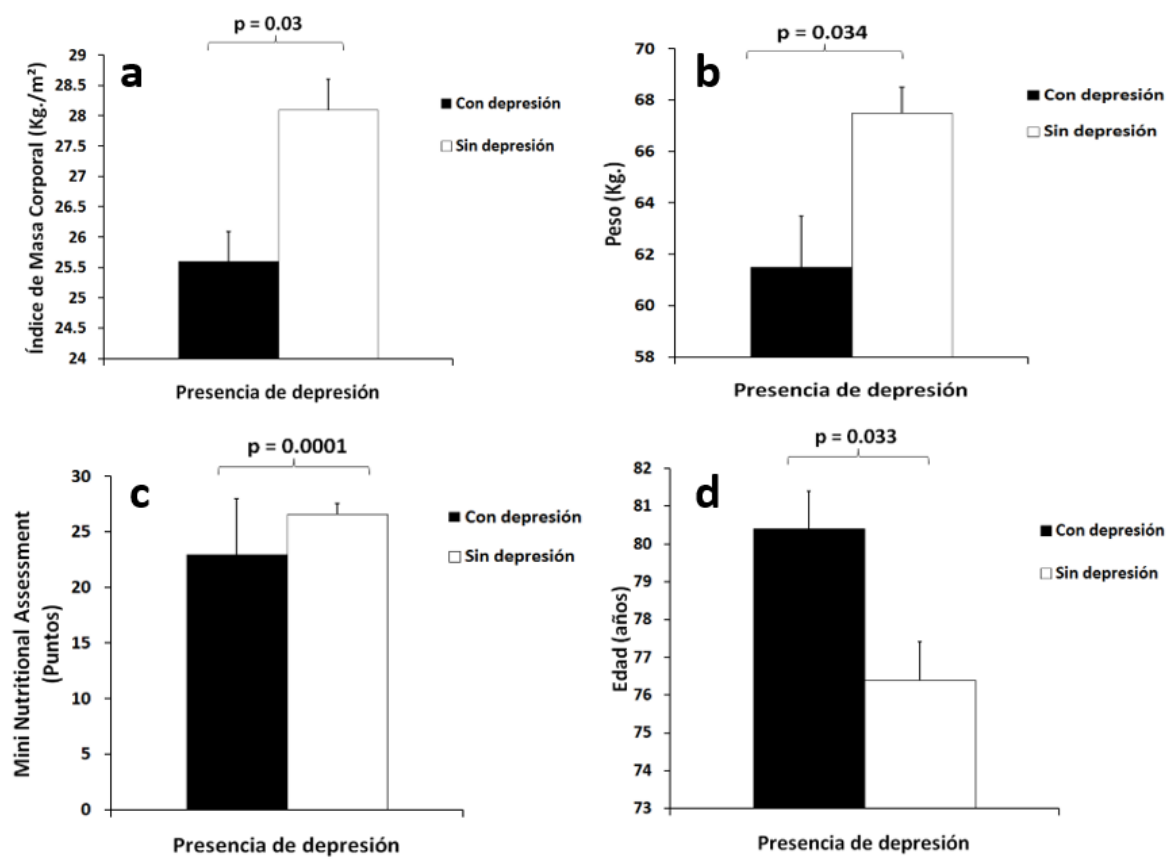

Figura 1. Antropometría y riesgo nutricio en presencia de depresión. Prueba estadística utilizada T-Student para muestras independientes. $(n=50)$. Se consideró significativo a partir de una $\mathrm{p}<0.5$

\section{Bajo peso y depresión}

De acuerdo con el análisis de correlación de Pearson, se observó una relación inversamente proporcional entre el MNA y la presencia de depresión $(\mathrm{r}=-0.528$, $\mathrm{p}=0.0001$ ); es decir que a mayor puntaje del MNA, menor es la presencia de depresión. Una persona con mejor estado de nutrición tendrá menor presencia de sintomatología depresiva. Los resultados se pueden observar en la tabla 3 y figura 2.

\begin{tabular}{lccc}
\hline & \multicolumn{1}{c}{$\begin{array}{c}\text { Variable regresora } \\
\text { Escala de Depresión }\end{array}$} & & \\
& $\begin{array}{c}\text { Esiable } \\
\text { Geriátrica de Yesavage (puntos) }\end{array}$ & & \\
\hline Talla $(\mathrm{cm})$ & -0.054 & 0.708 \\
Peso $(\mathrm{Kg})$ & -0.148 & 0.305 \\
IMC $\left(\mathrm{Kg} / \mathrm{m}^{2}\right)$ & -0.207 & 0.153 \\
Mini Nutritional Assessment & & $\mathbf{0 . 5 2 8}$ & $\mathbf{0 . 0 0 0 1}$ \\
(puntos) & & & \\
\hline
\end{tabular}

Tabla 3. Correlación de variables antropométricas con la presencia de depresión Prueba utilizada: Correlación de Pearson, $(n=50)$. Se consideró significativa con una $\mathrm{p}<0.05$. 


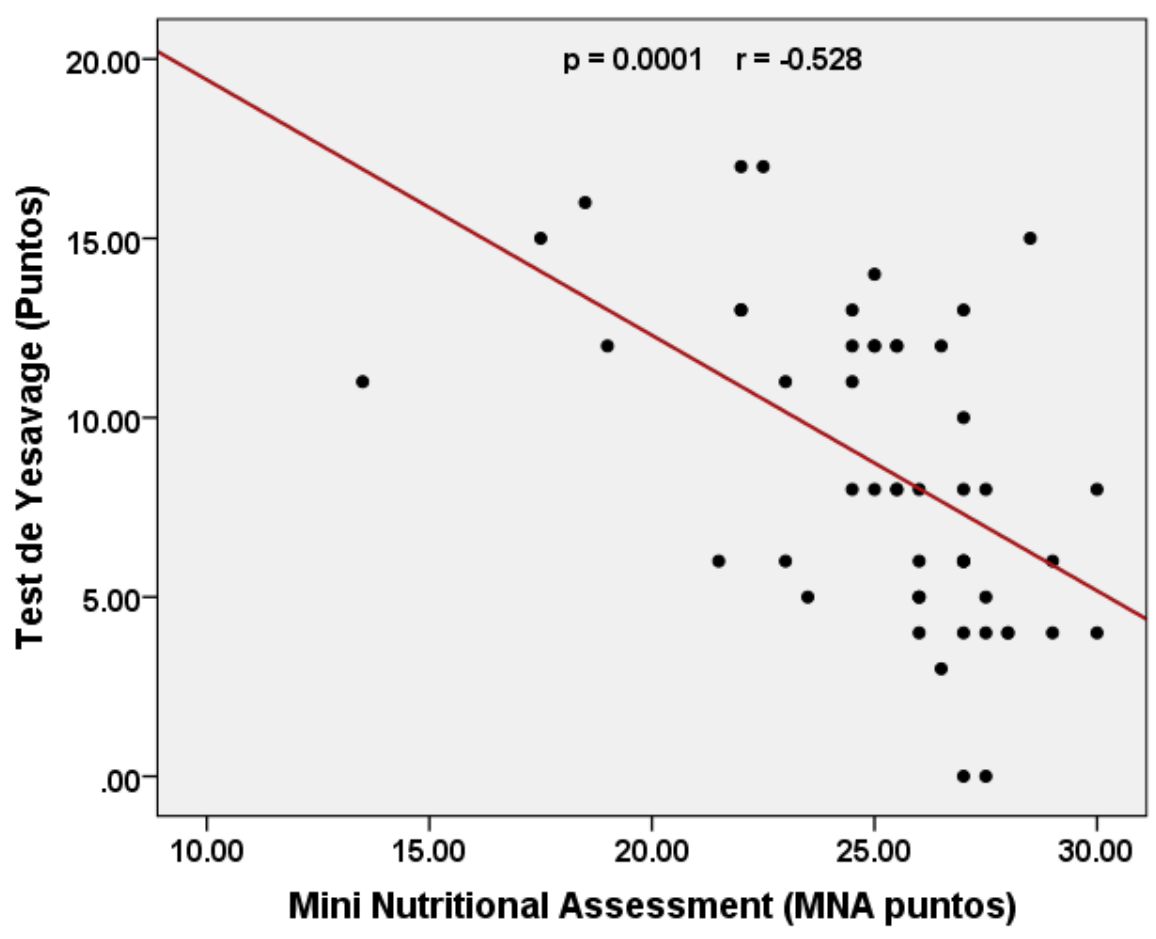

Figura 2. Correlación entre riesgo nutricional y depresión. Prueba estadística utilizada: Correlación de Pearson. $(\mathrm{n}=50)$.

\section{DISCUSIÓN}

La prevalencia de desnutrición o de riesgo nutricional en la población de adultos mayores dependerá de acuerdo a la situación actual de la persona; es decir, existen cambios importantes en la prevalencia si la población es ambulatoria o hospitalizada. En este caso, nuestros datos mostraron resultados similares a los observados por Cabrera y cols. [Malnutrición: (2\% vs $1.8 \%$ ) y riesgo nutricional $(22 \%$ vs $19.8 \%)$ ], esto fue obtenido en ambas poblaciones ambulatorias. Sin embargo, es muy variado de acuerdo a la ciudad. En México se obtuvo una prevalencia del $15.5 \%$ sin malnutrición, el $72.2 \%$ en riesgo y el $11.3 \%$ con desnutrición; esto en población ambulatoria e institucionalizada. ${ }^{8}$ Por otro lado, la presencia de depresión fue mayor en mujeres (45\%) esto puede ser explicado debido a la llegada de la menopausia y la falta de estrógenos, como lo describen Vega-Rivera y cols., que nos hablan sobre la modulación por parte de los estrógenos sobre el sistema noradrenérgico, el cual pudiera inducir efectos antidepresivos, además de los efectos propios de los estrógenos a nivel conductual. ${ }^{10,18}$ Se ha observado que en mujeres de 45 a 64 años de edad, menopáusicas y con un mayor IMC, esto se asocia con un menor puntaje en síntomas depresivos. Esto puede deberse a que las mujeres con mayor tejido adiposo tienen concentraciones de estrógenos que aportan cierta protección contra el desarrollo de síntomas depresivos. ${ }^{19}$

En cuanto al peso, se ha reportado que las condiciones psiquiátricas como la depresión y la demencia influyen en la pérdida de peso de un $9 \%$ a un $24 \% .{ }^{20}$ En otros estudios se ha observado que la depresión, la falla renal, y la diabetes mellitus 
estuvieron significativamente relacionadas con la pérdida de peso. ${ }^{21}$ El IMC y/ o un peso bajo y su relación con la presencia de depresión, fue estudiada por Kvamme, ${ }^{22}$ observando que las personas con obesidad no presentaban depresión. Cabe mencionar que nuestros resultados coinciden con estos datos. Sin embargo, diversos estudios de Wit L, Zhao y cols. han reportado en adultos con obesidad la presencia de diversos síntomas depresivos. No obstante, el IMC no parece ser una condicionante para que se presente en ambos estados nutricionales, tanto en desnutrición como en obesidad. Cabe resaltar que esto fue encontrado en población de diversas edades, lo cual pudo haberse debido a factores ajenos al IMC. ${ }^{23,24,25}$

Sin embargo, otro estudio en Corea reportó que, en población geriátrica, la depresión se presenta en personas con bajo peso y con obesidad, pero el nivel de depresión es menor en personas con sobrepeso y con peso normal. ${ }^{26}$ $\mathrm{Al}$ utilizar otra herramienta para evaluar la depresión, se ha reportado que el riesgo nutricional ha sido mayor en las personas deprimidas, incluyendo otros síndromes geriátricos como trastornos del sueño y caídas. ${ }^{17}$

Por otro lado, factores como el deterioro fisiológico, problemas con la masticación, deglución y una baja ingesta dietética pueden contribuir a enfermedades carenciales, como el caso de la anemia, ya sea por déficit de folatos o cobalamina (Vitamina B12) -micronutrimentos que participan en diversas funciones a nivel neurológico-, contribuyendo esto a la aparición de síntomas depresivos. Ávila y cols. reportan una ingesta baja en el consumo de productos lácteos, carne, pescado, aves, frutas y verduras en adultos mayores con síntomas depresivos. ${ }^{27}$ Sin embargo, en otro estudio realizado por Heuberger en una comunidad rural, no se presentaron diferencias significativas en el consumo de hierro, folatos, vitamina B12 y omega-3, entre las personas deprimidas y las que no presentaban depresión. ${ }^{16}$

La población mayor de 60 años de la ciudad de Aguascalientes presentó una asociación con los resultados obtenidos del instrumento de valoración de riesgo nutricional del MNA y el puntaje de la Escala de Depresión Geriátrica de Yesavage; es decir, los adultos mayores con depresión presentan un estado nutricional en riesgo o de malnutrición. Nuestros datos arrojan resultados similares a estudios aplicados en diversas poblaciones (EUA, Irán, México, Perú), tanto en comunidad, como en adultos mayores institucionalizados. ${ }^{28,29,30,31} \mathrm{No}$ obstante, una de las limitaciones del estudio es el tamaño reducido de la muestra, por lo que estos resultados deben ser considerados con discreción y animar a continuar con dicha brecha de investigación. Finalmente, se observó el papel de la longevidad en la presencia de depresión; una mayor edad condicionará la presencia de depresión, como es reportado en diversos estudios en México de Badillo y cols. ${ }^{30}$ Como sabemos, una mayor edad aumenta el riesgo de desarrollar desnutrición, ${ }^{12}$ traduciendo esto en deficiencias que producen deterioro físico y mental, disminuyendo la calidad de vida. 


\section{CONCLUSIÓN}

Es relevante dar un seguimiento en estudios posteriores a la modulación del estado nutricional en relación con el estado de ánimo del adulto mayor, lo cual denota la necesidad que existe de que el adulto mayor reciba una atención integral, promoviendo ambientes que favorezcan el estado emocional, así como una evaluación nutricional oportuna y adecuada, mejorando las expectativas y calidad de vida en esta etapa.

\section{Conflicto de intereses}

Los autores declaran que no hay conflicto de intereses con la utilización de datos y la investigación llevada a cabo, así como la publicación de los resultados.

\section{Referencias}

1. Porter T. Statement in support of the WHO World Report on Ageing and Health. WHO, HelpAge. 2016; 9:1-2.

2. INAPAM. Estadísticas sobre adultos mayores en México. INEGI, Censo de población y vivienda. 2016; 9(22): 1-4.

3. Peña Solano DM, Herazo Dilson MI, Calvo Gómez JM. Depresión en ancianos. RevFacMed. 2009; 57(4):347-355.

4. Sotelo Alonso I, Rojas Soto JE, Sánchez Arenas C, Irigoyen Coria A. La depresión en el adulto mayor: una perspectiva clínica y epidemiológica desde el primer nivel de atención. Arch Med Fam. 2012; 14(1): 5-13.

5. Rodríguez-Domínguez Z, Casado-Méndez P, Molero-Segrera M. et al. Evaluación del cuestionario de Yesavage abreviado versión española en el diagnóstico de depresión en población geriátrica. Rev Hos Psi Hab. 2015; 12(3):1-9.

6. Vafaeri Z, Mokhtari H, Sadooghi Z, Meamar R, Chitsaz A, Moeini M. Malnutrition is associated with depression in rural elderly population. J Res Med Sci. 2013; 18 (S1): S15-S19

7. Aguilar Navarro S, Ávila Funes JA. La depresión: particularidades clínicas y consecuencias en el adulto mayor. Gac Med Méx. 2007; 143(2): 141-148

8. Cabrera MA, Mesas AE, Garcia AR, de Andrade SM. Malnutrition and depression among community-dwelling elderly people. J Am Med Dir Assoc. 2007; 8(9): 582-584.

9. Pérez Cruz E, Lizárraga Sánchez C, Martínez Esteves MR. Asociación entre desnutrición y depresión en el adulto mayor. Nutr Hosp. 2014; 29(4): 901-906.

10. Ruiz de Chávez Ramírez DA. Zegbe J Sánchez Morales FM. Castañeda Iñiguez MS. Depresión en adultos mayores atendidos en instituciones públicas de salud en Zacatecas. Revista de Educación y Desarrollo. 2014; (1): 73-78.

11. Casimiro C, García de Lorenzo A, Usán L. Evaluación del riesgo nutricional en pacientes ancianos ambulatorios. Nutr. Hosp. 2001; 16(3): 97-103.

12. Smoliner C, Norman K, Wagner KH, Hartig W, Lochs H, Pirlich M. Malnutrition and depression in the institutionalised elderly. Br J Nutr. 2009; 102: 1663-1667.

13. Calderón-Reyes ME, Ibarra-Ramírez F, García, et al. Evaluación nutricional comparada del adulto mayor en consultas de medicina familiar. Nutr Hosp. 2010; 25(4): 669-675. 
14. Méndez Estévez E, Romero Pita J, Fernández Rodríguez MJ, Troitiño Álvarez P, García Dopazo S, Jardón Blanco M, et al. ¿ Tienen nuestros ancianos un adecuado estado nutricional? ¿Influye su institucionalización? Nutr Hosp. 2013; 28(3): 903-913.

15. Kronfly Rubiano E, Rivilla Frías D, Ortega Abarca I, et al. Riesgo de depresión en personas de 75 años o más, valoración geriátrica integral y factores de vulnerabilidad asociados en atención primaria. Aten primaria. 2015; 47(10): 616-625.

16. Heuberger $\mathrm{R}$, Wong $\mathrm{H}$. The association between depression and widowhood and nutritional status in older adults. Geriatr Nurs. 2014 35: 428-433.

17. Saka B, Kaya O, Bahan Ozturk G, Erten N, Akif Karan M. Malnutrition in the elderly and its relationship with other geriatric syndromes. Clin Nutr. 2010; 29(6):745-748.

18. Vega Rivera N, López Ruvalcaba C, Páez Martínez N, et al. Interacción estrógenosnoradrenalina en la depresión. Salud Ment. 2013; 36(4): 331-336.

19. Jasienska G, Ziomkiewicz A y cols. Body mass, depressive symptoms and menopausal status: An examination of the "Jolly fat" hypothesis. Women's health issues journal. 2005; 3(15): 145-151

20. Gaddey L H, Holder K. Unintentional weight loss in older adults. Am Fam Physician. 2014; 89(9): 718-722.

21. Tamura BK, Bell CL, Masaki KH. Amella EJ. Factors associated with weight loss, low BMI, and malnutrition among nursing home patients: a systematic review of the literature. Jamda. 2013 14: 649-655.

22. Kvamme JM, Gronli O, Florholmen J, Jacobsen BK. Risk of malnutrition is associated with mental health symptoms in community elderly men and women: The Tromso Study. BMC Psychiatry. 2011; 11(112):1-8.

23. M de Wit L, Van Straten A, Van Herten M, Penninx B WJH, Cuijpers P. Depression and body mass index, a u-shaped association. BMC Public Health. 2009; 9(14): $1-6$.

24. Zhao G, Ford ES, Li C, Tsai J, Dhingra S, Balluz LS. Waist circumference, abdominal obesity, and depression among overweigth and obese U.S. adults: national health and nutrition examination survey 2005-2006. BMC Psychiatry. 2011; 11 (130):1-9.

25. German L, Feldblum I, Bilenko N, Castel H, Harman-Boehm I, Shahar DR. Depressive symptoms and risk for malnutrition among hospitalized elderly people. J Nutr Health Aging. 2008; 12(5): 313-318.

26. Noh JW, Kwon YD, Park J, Kim J. Body mass index and depressive symptoms in middle aged and older adults. BMC public health. 2015; 15(310): 1-7.

27. Ávila-Funes JA, Garant MP, Aguilar Navarro S. Relación entre los factores que determinan los síntomas depresivos y los hábitos alimentarios en adultos mayores de México. Rev Panam Salud Pública. 2006; 19(5):321-30.

28. Keshavarzi S, Ahmadi SM, Lankarani KB. The impact of depression and malnutrition on health-related quality of life among the elderly iranians. Glob J Health Sci. 2015; 7(3): 161-170.

29. Soundararajan AS, Mathew AC, Nanjuudan R, Ganesh A. Association of geriatric syndromes with malnutrition among elderly. Int J Med Res Health Sci. 2017; 6(5): $14-18$ 
30. Durán Badillo T, Aguilar RM, Martínez ML, Rodríguez T, Gutiérrez G, Vázquez L. Depresión y función cognitiva de adultos mayores de una comunidad urbana marginal. Enferm. univ. 2013; 10(2): 36-42.

31. Contreras AL, Ángel Mayo GV, Romaní DA, Silvana Tejada G, et al. Malnutrición del adulto mayor y factores asociados en el distrito de Masma Chicche, Junín, Perú. Rev Med Hered. 2013; 24(3): 186-191. 